\title{
Intracellular Location of the SOX9 Protein in Breast Disease
}

\author{
Gary L. Bratthauer* and Tuyethoa N. Vinh
}

Department of Gynecologic and Breast Pathology, Armed Forces Institute of Pathology, Washington, DC 20306-6000, USA

\begin{abstract}
SOX9, an important factor for testis determination and chondrogenesis, was previously detected in the nucleus of testicular sertoli cells and chondrocytes but was found in only a subset of ovarian sertoli cell tumors, carcinoid tumors, and endometrioid carcinomas, and occasionally only in the cytoplasm of those cells. SOX9 has also been detected in metaplastic carcinomas of the breast exhibiting chondroid differentiation so it became of interest to examine SOX9 in other breast carcinomas and normal breast. Normal breast lobules were studied immunohistochemically for SOX 9 along with examples of metaplastic breast carcinoma exhibiting chondroid differentiation, lobular neoplasia (ALH/LCIS), invasive lobular carcinomas, and ductal carcinomas (invasive and in situ). SOX9 was identified solely in the nucleus in over $90 \%$ of the normal breast epithelial cells examined. In 10 of 12 breast carcinomas exhibiting chondroid metaplasia, and in 15 of 16 invasive lobular carcinomas, SOX9 was present primarily in the nucleus much like that seen in normal breast epithelium. In 14 of 20 invasive ductal carcinomas, however, it was missing or identified mainly in the cytoplasm of the malignant cells. The functional importance of SOX9 in the breast is unknown although transcription factors involved with the development of certain tissues may have similar capabilities in other organs. The retention of SOX9 in the nucleus of metaplastic or lobular carcinomas could indicate some functional expression of this protein in these tumors with an even more compelling reason why it is often restricted to the cytoplasm, or missing, in ductal carcinomas of the breast.
\end{abstract}

\section{INTRODUCTION}

SOX9, the SRY (sex-determining region on the $\mathrm{Y}$ chromosome)-type high-mobility-group box transcription factor 9, is known to be involved in testis formation and chondrogenesis. SOX9 was first identified as a testisdetermining factor although many downstream target genes have yet to be identified [1]. SOX9 was found to have a male specific role in sex determination and was shown to be specific to the sertoli cell lineage $[2,3]$. SOX9 expression also correlates with chondrocyte gene expression involving the production of collagen type II while having a major role in chondrogenesis and skeletal development [4-6]. Using an antibody recognizing SOX9 we detected the protein in the nucleus of testicular sertoli cells and chondrocytes. These cells and the nuclear location are in keeping with the described function of this transcription factor.

Mutations in the SOX9 gene can lead to human disease. Changes in SOX9 resulting in decreased DNA binding ability were shown to be present in patients with the cartilage disease campomelic dysplasia [7]. Also, SOX9 was shown to be present in over $95 \%$ of the nuclei of chondrosarcomas [8]. In our previous study, SOX9 was identified, inconsistently, in a subset of sertoli cell tumors of the ovary [9]. However, a subset of endometrioid carcinomas and carcinoid tumors of the ovary were also found to contain this protein. Moreover, other carcinomas were additionally found to be reactive with the SOX9 antibody but only in the cytoplasm or on the

*Address correspondence to this author at the Department of Gynecologic and Breast Pathology, Armed Forces Institute of Pathology, Washington, DC 20306-6000, USA; Tel: 202-782-1600; Fax: 202-782-3939;

E-mail: bratthauer@afip.osd.mil cytoplasmic membrane. Since SOX9 functions as a transcription factor, the nuclear location of the protein in certain cells was an expected finding, but the solely cytoplasmic or membranous location seen in some malignant cells was unexpected.

Because of SOX9's presence in the nucleus or the cytoplasm of some ovarian carcinomas it became of interest to analyze breast carcinomas as well; a gland also under hormonal influence in the female. Recently, a report by Kusafuka et al. showed SOX9 to be present in 4 out of 4 cases of a subset of breast carcinomas that are metaplastic with cartilaginous features [10]. Metaplastic breast carcinoma is a rare tumor with a worse prognosis than typical breast cancer [11]. Since some metaplastic breast carcinomas exhibit chondroid differentiation it is reasonable to think that SOX9 might be present. We wanted to expand on this finding by examining more metaplastic breast carcinomas along with ductal and lobular carcinomas of the breast for the presence of SOX9 in order to determine if a possible role for SOX9 exists in the formation of these and other lesions.

\section{MATERIALS AND METHODOLOGY}

Formalin-fixed, paraffin-embedded, samples of breast carcinoma were obtained from the files of the Armed Forces Institute of Pathology (AFIP) after Institution Review Board (IRB) approval and studied immunohistochemically using the SOX9 antibody. The lesions examined, in addition to normal terminal duct lobular units, included 20 invasive ductal carcinomas, 18 examples of ductal carcinoma in situ (DCIS), 16 invasive lobular carcinomas, 23 examples of lobular neoplasia (ALH/LCIS) and 12 specimens of metaplastic breast carcinoma exhibiting chondroid 
differentiation only. Lobular lesions were defined as having the morphologic appearance of lobular breast disease and being E-cadherin negative.

Immunohistochemical stains were performed as previously described [9, 12]. Essentially, a polyclonal antiserum reactive against SOX9 (Chemicon Inc., Temecula, CA, USA), was detected on tissue using an $\mathrm{ABC}$ technique (Vector Laboratories, Burlingame, CA, USA) following pretreatment in a pressure cooker with Reveal (Biocare Medical, Concord, CA, USA). Normal rabbit immunoglobulins assayed on duplicate sections served as negative controls. A known SOX9 positive section of normal testis demonstrating sertoli cells and a section of cartilage were used as positive controls on the assay. All sections were reviewed for intensity and distribution of reaction product. Results were tabulated for each final diagnosis by intracellular location and recorded qualitatively. A positive result was determined to represent at least $30 \%$ of the tumor cells being reactive.

\section{RESULTS}

The results are listed in Table 1. SOX9 was strongly identified solely in the nucleus in over $90 \%$ of the normal breast epithelial cells examined in all of the samples where normal foci existed whether close to, or away from, the lesion studied (Fig. 1a, b). All negative controls were nonreactive. Positive controls demonstrated a nuclear reaction product in both sertoli cells of the testis and chondrocytes. In
10 of the 12 breast carcinomas exhibiting chondroid metaplasia SOX9 was found only in the nucleus much like that seen in normal breast epithelium, testicular sertoli cells, and chondrocytes (Fig. 1c, d). The protein was identified in most of the tumor cells with an increase in intensity associated with those cells fully exhibiting chondroid differentiation. In the remaining two cases the majority of the tumor cells were non-reactive with anti-SOX9.

Substantial nuclear reactivity was also seen in most of the invasive lobular carcinomas tested (Fig. 2a, b). SOX9 was expressed in the nucleus in 15 out of the $16(>90 \%)$ invasive lobular carcinoma samples. Interestingly, in 14 of the 20 invasive ductal carcinomas examined, SOX9 was either missing or identified mainly in the cytoplasm of the malignant cells (Fig. 2c, d). When present, SOX9 was found in the nucleus in only $30 \%$ of these lesions, whereas $65 \%$ contained SOX9 predominantly in the cytoplasm.

The in situ lesions were similar to their invasive counterparts in intracellular location of SOX9 with almost $80 \%$ nuclear expression seen in cases of lobular neoplasia (ALH/LCIS) and 55\% cytoplasmic expression seen in the DCIS samples (Fig. 3). In DCIS, predominant nuclear expression was seen in less than $40 \%$ of the samples, and in lobular neoplasia (ALH/LCIS) SOX9 was either missing or present in the cytoplasm in only a little over $20 \%$ of the samples.
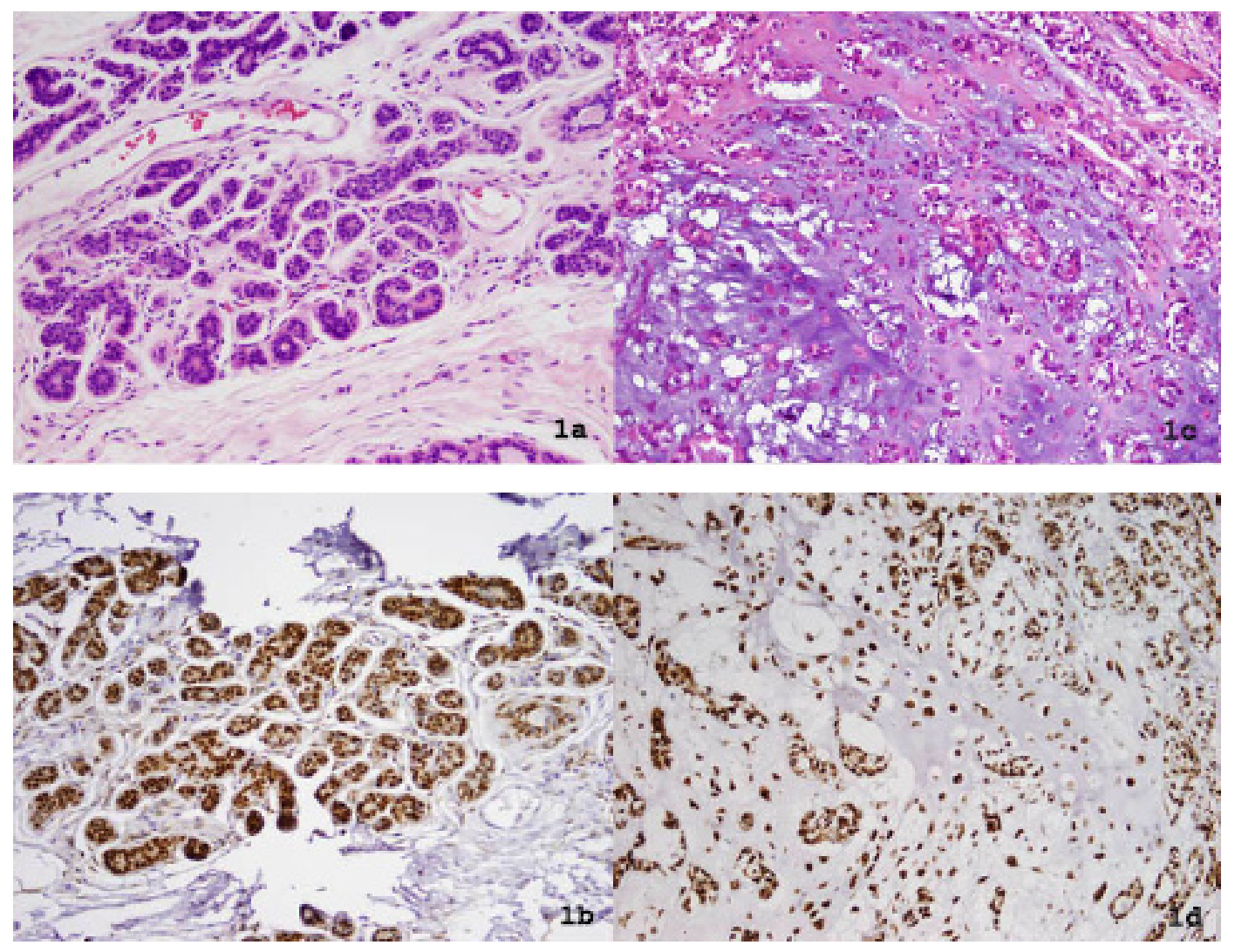

Fig. (1). (a) Hematoxylin and Eosin stain of normal breast. (b) Immunohistochemical demonstration of SOX9 in normal breast. Note location (brown) in the nuclei of the luminal breast epithelial cells. (c) Hematoxylin and Eosin stain of a metaplastic breast carcinoma exhibiting chondroid differentiation. (d) Immunohistochemical demonstration of SOX9 in the nuclei of the tumor cells showing chondroid differentiation in this breast carcinoma. X200. 

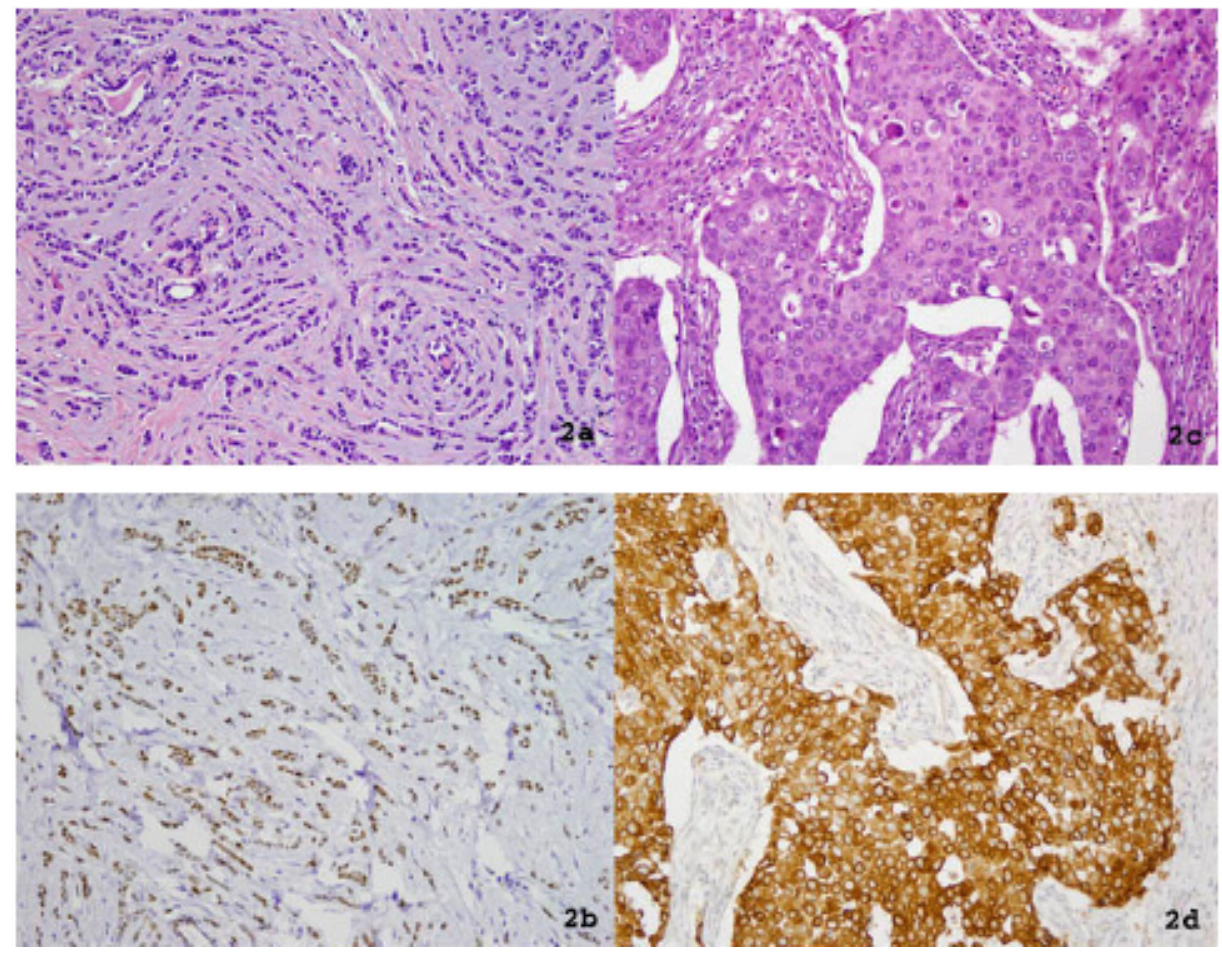

Fig. (2). (a) Hematoxylin and Eosin stain of an invasive lobular carcinoma of the breast. (b) Immunohistochemical demonstration of SOX9 in the nuclei of the tumor cells in this lobular breast carcinoma. (c) Hematoxylin and Eosin stain of an invasive ductal carcinoma of the breast. (d) Immunohistochemical demonstration of SOX9 in the cytoplasm of the cells in this ductal breast carcinoma.

There was a little expression overlap seen with $31 \%$ of all of the lobular lesions and $54 \%$ of all of the ductal lesions with SOX9 predominantly expressed in the nucleus also demonstrating some cytoplasmic expression while none of the ductal or lobular lesions expressing SOX9 predominantly in the cytoplasm demonstrated any nuclear expression.

Whether SOX9 was missing or found only in the cytoplasm of invasive ductal carcinomas, the adjacent normal cells were shown to express the SOX9 protein in the nucleus (Fig. 4a, b). In some cases, where available, it appeared that the level of SOX9 cytoplasmic reactivity seen in DCIS increased in the adjacent invasive carcinoma component (Fig. 4c). Also, benign proliferative glands like Flat Epithelial Atypia were often observed to have lost luminal cell SOX9 expression while maintaining SOX9 in the myoepithelial cell layer (Fig. 4d).

\section{DISCUSSION}

Although the expression of SOX9 has been shown to be important for a number of organ systems its aberrant expression can lead to indicators of disease [13]. Since sex hormones have been shown to play a role in SOX9 expression it is not surprising that its expression has been noted in some ovarian tumors $[9,14]$. SOX9 was also observed to be activated in basal cell carcinomas of the skin, gastric as well as colorectal cancers, and in prostate cancer cells refractory to hormone therapy it was shown to enhance tumor cell proliferation [15-18]. Therefore, despite the necessity of SOX9 involvement in the development of normal tissues it seems that it might also play a role in disease under certain conditions. The ultimate effects of SOX9 depend upon the genes on which it acts and the accessibility of those genes in various cells.
SOX9 has been implicated in breast disease with activity in breast cancer cell lines suggesting it is stimulated by agonists for the retinoic acid receptor [19]. These receptor ligands inhibit the growth of normal and carcinoma cell lines. This growth inhibition is concomitant with a stimulation of SOX9 expression and the introduction of a dominant negative SOX9 was recently shown to inhibit the effect of retinoic acid induced expression [20]. While SOX9 has been shown as a collagen type II stimulator in chondrocytes and has a role in male differentiation in the testis, its role is largely unknown in mediating growth inhibition in breast tumors. Its up-regulation by retinoic acid receptor agonists suggests it may play a role in normal breast physiology [19]. A recent report identified SOX9 in the nucleus of 4 out of 4 metaplastic breast carcinomas exhibiting chondroid differentiation [10]. There was little mentioned about the presence of SOX9 or the intracellular location of the protein in normal breast epithelial cells. In this present study we found nuclear expression of SOX9 in the majority of the studied metaplastic breast carcinomas exhibiting chondroid differentiation. We also found, however, consistent expression of SOX9 in over $90 \%$ of normal breast epithelial cell nuclei and in the nuclei of a vast majority of lobular carcinomas as well. Kusafuka et al. found less nuclear expression in the areas of typical carcinoma in their 4 metaplastic breast carcinoma cases [10]. Likewise, in our ductal breast carcinomas that did not exhibit any metaplastic features, SOX9 was occasionally absent. However, in almost two thirds of these ductal breast lesions, SOX9 was located in the cytoplasm, not the nucleus, of the malignant cells. It is indeed interesting whenever the intracellular expression of regulatory proteins is altered in abnormal cells. 

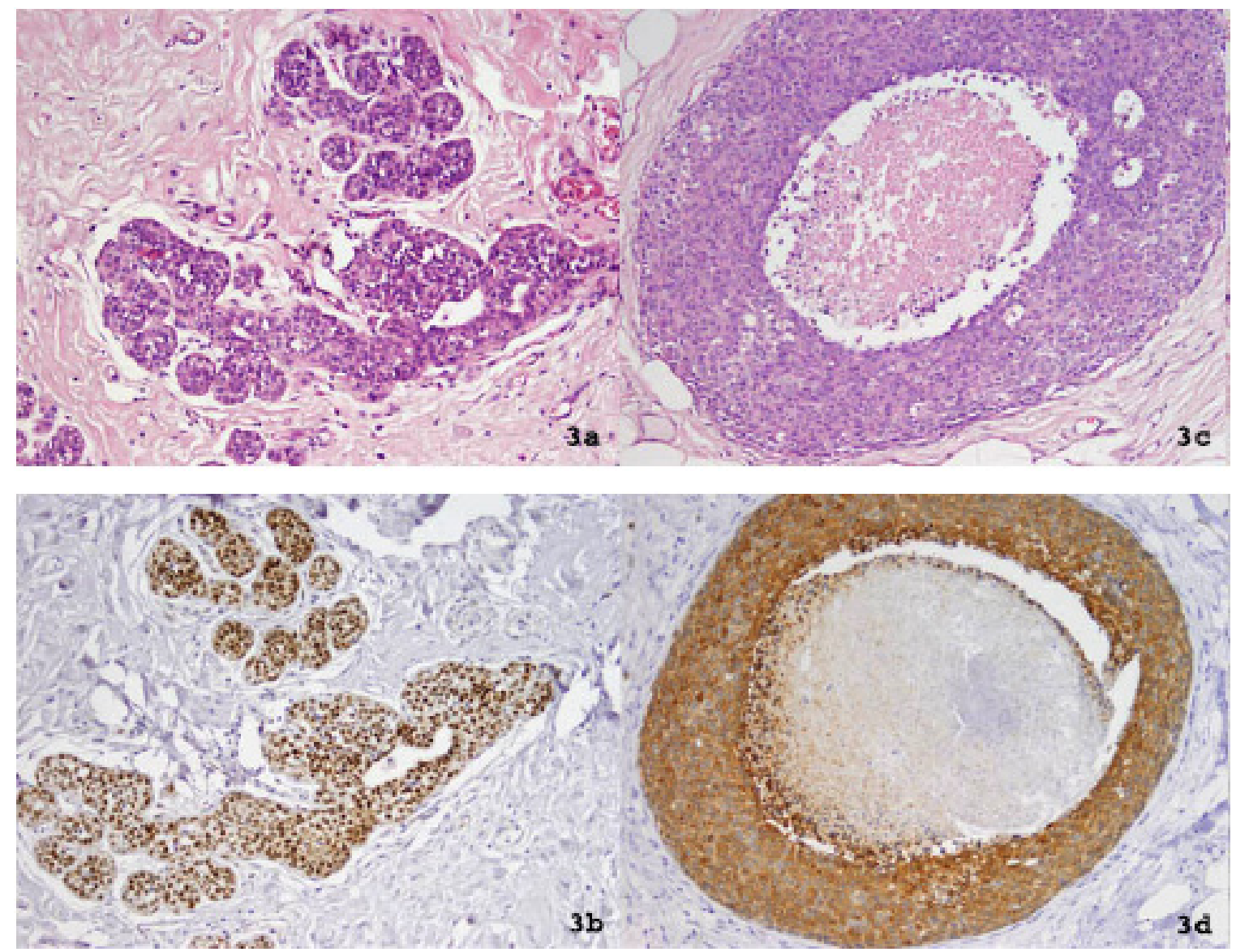

Fig. (3). (a) Hematoxylin and Eosin stain of lobular neoplasia (ALH/LCIS) of the breast. (b) Immunohistochemical demonstration of SOX9 in the nuclei of the cells in this breast lesion. X200. (c) Hematoxylin and Eosin stain of ductal carcinoma in situ (DCIS) of the breast. (d) Immunohistochemical demonstration of SOX9 in the cytoplasm of the cells in this in situ carcinoma. X200.

Table 1. Expression and Intracellular Distribution of SOX9 in Breast Lesions

\begin{tabular}{|l|c|c|c|c|}
\hline \multirow{2}{*}{\multicolumn{1}{c|}{ Diagnosis }} & \multicolumn{4}{c|}{ SOX9 Predominant Location } \\
\cline { 2 - 5 } & $\begin{array}{c}\text { Total } \\
\text { Samples }\end{array}$ & Nuclear & Cytoplasmic & Negative \\
\hline \hline LN (ALH/LCIS) & 23 & $18(78 \%)$ & $3(13 \%)$ & $2(9 \%)$ \\
\hline Invasive Lobular CA & 16 & $15(94 \%)$ & $1(6 \%)$ & 0 \\
\hline DCIS & 18 & $7(39 \%)$ & $10(56 \%)$ & $1(5 \%)$ \\
\hline Invasive Ductal CA & 20 & $6(30 \%)$ & $13(65 \%)$ & $1(5 \%)$ \\
\hline Metaplastic Carcinoma & 12 & $10(83 \%)$ & 0 & $2(17 \%)$ \\
\hline $\begin{array}{l}\text { Normal Breast } \\
\text { Epithelium }\end{array}$ & 80 & $80(100 \%)$ & 0 & 0 \\
\hline
\end{tabular}

LN: lobular neoplasia; ALH: atypical lobular hyperplasia; LCIS: lobular carcinoma. In situ; DCIS: ductal carcinoma in situ; CA: carcinoma.

Metaplastic carcinomas of the breast are rare tumors often with P53 mutations [21]. SOX9 normally regulates the transformation of mesenchymal cells to chondrocytes in the formation of bone and cartilage. Since some metaplastic carcinomas demonstrate chondrocytic differentiation it is reasonable to assume that SOX9 may be active in these cells. Interactions with beta-catenin are involved in the process of chondrogenesis and beta-catenin has been shown to be present in different locations in normal and abnormal breast epithelial cells $[22,23]$. SOX9 has been shown to be expressed in colon carcinoma cell lines where a Wnt pathway beta-catenin-TGF4 complex is required possibly helping to keep a Wnt dependent progenitor phenotype [24]. It is already known that genetic changes in the Wnt pathway genes are common events in metaplastic carcinomas of the breast [25]. SOX9 is also down-regulated when there is increased collagenase activity [26]. During fracture healing, the rise and fall of SOX9 expression correlates with specific expression of the cartilage proteins, collagen type II, and retinoic acid sensitive protein [27]. It stands to reason therefore that an active SOX9 can have the ability to stimulate the formation of cartilage under the right conditions. The cartilage formation may occur through a retained, active, SOX9 coupled with other conditions allowing for the transcription of genes perhaps identical to the ones seen in mesenchymal cells undergoing chondrogenesis. In this case, SOX9 may be functioning in a normal way for mesenchymal cell differentiation. In the mouse, prostate tumor endothelial cells can demonstrate chondrogenic differentiation when accompanied by the upregulation of SOX9 [28]. Hypoxia up-regulates the expression of SOX9 through HIF-1alpha in mesenchymal cells that differentiate to chondrocytes [29]. Perhaps an increased state of hypoxia present within certain metaplastic breast cancers allow for this up-regulation as well. Also, 

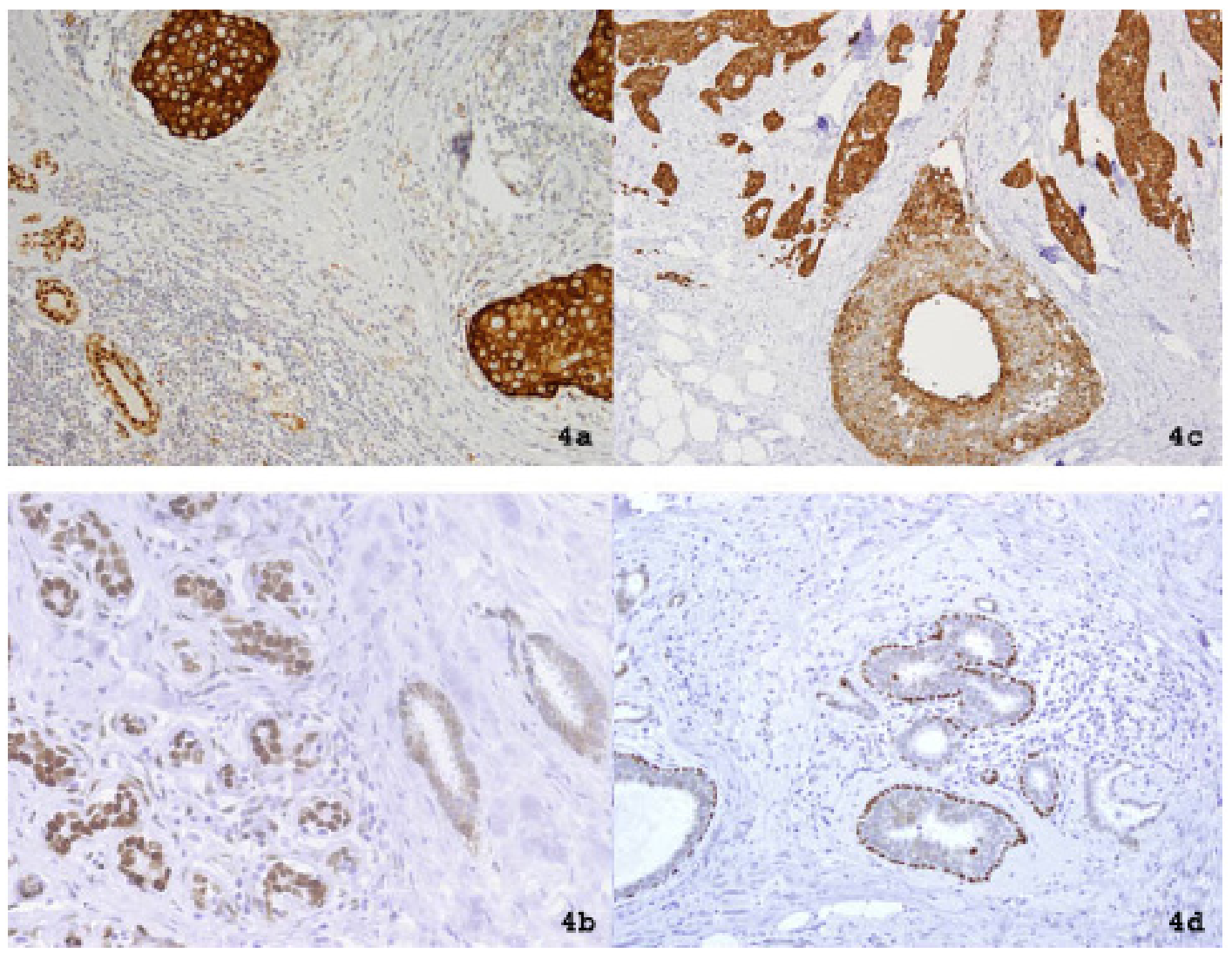

Fig. (4). (a) SOX9 expression in the cytoplasm of invasive ductal carcinoma of the breast adjacent to normal glands. X200. (b) Lack of SOX9 expression in tubular carcinoma of the breast adjacent to normal glands. X400. (c) Increased intensity of SOX9 cytoplasmic expression in breast invasive ductal carcinoma over that expressed in the adjacent DCIS. X100. (d) SOX9 expression in the myoepithelial but not the luminal cell layer of Flat Epithelial Atypia adjacent to tubular carcinoma of the breast. X200.

with metaplastic carcinomas being generally of the basal phenotype, and with most of the myoepithelial cells demonstrating expression of SOX9, it is possible that other types of metaplastic breast carcinomas may express nuclear SOX9 regardless of cartilage formation.

SOX9 shifts from cytoplasmic to nuclear location at the time of testis differentiation and anti-mullerian hormone expression [30]. Since an active SOX9 has been functionally linked to testis differentiation, and a nuclear location has been seen for this protein in the testis, the nuclear location observed for SOX9 in normal breast epithelial cells could be indicative of an active SOX9. While SOX9 is capable of stimulating different genes in various cell types the genes involved in SOX9 mediated activity in the breast have yet to be determined. The presence of SOX9 in the nucleus of malignant lobular epithelial cells may indicate normal SOX9 functions in these cells, or may merely indicate the presence of a structural antigenicity having lost some function through malignancy. Since lobular lesions are also defined as luminal, the presence of SOX9 in the nucleus in these lesions demonstrates a luminal as well as a basal identity for this protein.

Of additional interest is the qualitative reduction of SOX9 expressed in the nucleus in most ductal carcinomas of the breast. While SOX9 was mostly detected in the nucleus of normal cells it was either missing or found only in the cytoplasm of most in situ or invasive ductal breast cancers. In bladder cancer the SOX9 DNA was shown to be hypermethylated, and thus down- regulated, and the hypermethylated state of SOX9 was shown to correlate with tumor grade progression and overall survival [31]. This evidence leads to speculation that perhaps the absence of active SOX9 in ductal breast carcinomas could be a factor in increasing epithelial cell growth. An examination of the mutational or methylation status of SOX9 in ductal breast carcinomas warrants further study along with a comparison to prognostic markers seen in other histologic and molecular grades of breast cancer. Much larger sample sizes and concentration on specific phenotypes of breast disease would allow for valid statistical correlations between these markers.

\section{CONCLUSION}

The nuclear location of SOX9 in normal cells of the breast suggests that it may play a role in normal breast epithelial cell maturation by targeting breast specific metabolic genes and the fact that it is missing or restricted to the cytoplasm in the majority of ductal breast carcinomas may indicate an alteration that prevents normal cell metabolism leading to disease. The retention of SOX9 in the nucleus of most lobular breast lesions perhaps is indicative of an active SOX9 either with the same functionality seen in normal cells or an aberrant one involving a lobular carcinoma specific pathway. In the rare case of metaplastic breast cancer cells that continue to express nuclear SOX9 leading to cartilage formation the switch to a more mesenchymal chondroid phenotype in these cells may occur 
through a novel availability of matrix forming SOX9 target genes.

\section{ACKNOWLEDGEMENT}

The opinions or assertions contained herein are the private views of the authors and are not to be construed as official, or as reflecting the views of the Department of the Army, or the Department of Defense.

\section{REFERENCES}

[1] Harley VR, Clarkson MJ, Argentaro A. The molecular action and regulation of the testis-determining factors, SRY (sex-determining region on the Y chromosome) and SOX9 [SRY-related highmobility group (HMG) box 9]. Endocr Rev 2003; 24: 466-87.

[2] Kent J, Wheatley SC, Andrews JE, Sinclair AH, Koopman P. A male-specific role for SOX9 in vertebrate sex determination. Development 1996; 122: 2813-22.

[3] Forjdman K, Harley VR, Pelliniemi LJ. SOX9 protein in rat sertoli cells is age and stage dependent. Histochem Cell Biol 2000; 113: 31-6.

[4] Kolettas E, Muir HI, Barrett JC, Hardingham TE. Chondrocyte phenotype and cell survival are regulated by culture conditions and by specific cytokines through the expression of SOX 9 transcription factor. Rheumatology (Oxford) 2001; 40: 1146-56.

[5] Lefebvre V, de Crombrugghe B. Toward understanding SOX9 function in chondrocyte differentiation. Matrix Biol 1998; 16: 52940.

[6] Wright E, Hargrave MR, Christiansen J, et al. The Sry-related gene SOX9 is expressed during chondrogenesis in mouse embryos. Nat Genet 1995; 9: 15-20.

[7] Meyer J, Sudbeck P, Held M, et al. Mutational analysis of the SOX9 gene in campomelic dysplasia and autosomal sex reversal: Lack of genotype/phenotype correlations. Hum Mol Genet 1997; 6: 91-8.

[8] Wherli BM, Huang W, De Crombrugghe B, Ayala AG, Czerniak B. SOX9, a master regulator of chondrogenesis, distinguishes mesenchymal chondrosarcoma from other small blue round cell tumors. Hum Pathol 2003; 34: 263-69.

[9] Zhao CQ, Bratthauer GL, Barner R, Vang R. Immunohistochemical analysis of SOX9 in ovarian sertoli cell tumors and other tumors in the differential diagnosis. Int J Gynecol Pathol 2007; 26: 1-9.

[10] Kusafuka K, Muramatsu K, Kasami M, et al. Cartilaginous features in matrix-producing carcinoma of the breast: four cases report with histochemical and immunohistochemical analysis of matrix molecules. Mod Pathol 2008; 21: 1282-92.

[11] Rauf F, Kiyani N, Bhurgri Y. Metaplastic carcinoma of the breast, an intriguing rarity. Asian Pac J Cancer Prev 2008; 7: 667-71.

[12] Bratthauer GL. The Avidin-Biotin Complex (ABC) method and other avidin-biotin binding methods. Immunocytochemical Methods and Protocols. (Methods in Molecular Biology 115). In: Javois LC, Ed. Totowa, NJ. Humana Press Inc., 1999; pp. 203-14.

[13] Hanley KP, Oakley F, Sugden S, Wilson DI, Mann DA, Hanley NA. Ectopic SOX9 mediates extracellular matrix deposition characteristic of organ fibrosis. J Biol Chem 2008; 283: 14063-71.

[14] Malki S, Bibeau F, Notarnicola C, et al. Expression and biological role of the prostaglandin D synthase/SOX9 pathway in human ovarian cancer cells. Cancer Lett 2007; 255: 182-93.
[15] Vidal VP, Ortonne N, Schedl A. SOX9 expression is a general marker of basal cell carcinoma and adnexal-related neoplasms. J Cutan Pathol 2008; 35: 373-9.

[16] Yasui W, Oue N, Sentani K, Sakamoto N, Motoshita J. Transcriptome dissection of gastric cancer: Identification of novel diagnostic and therapeutic targets from pathology specimens. Pathol Int 2009; 59: 121-36.

[17] Lu B, Fang Y, Xu J, et al. Analysis of SOX9 expression in colorectal cancer. Am J Clin Pathol 2008; 130: 897-904.

[18] Wang H, Leav I, Ibaragi S, et al. SOX9 is expressed in human fetal prostate epithelium and enhances prostate cancer invasion. Cancer Res 2008; 68: 1625-30.

[19] Afonja O, Raaka BM, Huang A, et al. RAR agonists stimulate SOX9 gene expression in breast cancer cell lines: Evidence for a role in retinoid-mediated growth inhibition. Oncogene 2002; 21: 7850-60.

[20] Muller P, Crofts JD, Newman BS, et al. SOX9 mediates the retinoic acid-induced HES-1 gene expression in human breast cancer cells. Breast Cancer Res Treat. 2009 March 26; [About 25 screens]. Available from: http://www.springerlink.com/content/523 $4 \mathrm{~g} 51445 \mathrm{n} 7 \mathrm{r} 118 /$

[21] Bellino R, Arisio R, D'Addato F, et al. Metaplastic breast carcinomas: pathology and clinical outcome. Anticancer Res 2003; 23: 669-73.

[22] Akiyama H, Lyons JP, Mori-Akiyama Y, et al. Interactions between SOX9 and beta-catenin control chondrocyte differentiation. Genes Dev 2004; 18: 1072-87.

[23] Dabbs DJ, Kaplai M, Chivukula M, Kanbour A, Kanbour-Shakir A, Carter GJ. The spectrum of morphomolecular abnormalities of the E-cadherin/catenin complex in pleomorphic lobular carcinoma of the breast. Appl Immunohistochem Mol Morphol 2007; 15: 260-66.

[24] Blache P, van de Wetering M, Duluc I, et al. SOX9 is an intestine crypt transcription factor, is regulated by the Wnt pathway, and represses the CDX2 and MUC2 genes. J Biol Chem 2004; 166: 3747.

[25] Hayes MJ, Thomas D, Emmons A, Giordano TJ, Kleer CG. Genetic changes of Wnt pathway genes are common events in metaplastic carcinomas of the breast. Clin Cancer Res 2008; 14: 4038-44.

[26] Tchetina EV, Squires G, Poole AR. Increased type II collagen degradation and very early focal cartilage degeneration is associated with upregulation of chondrocyte differentiation related genes in early human articular cartilage lesions. J Rheumatol 2005; 32: 876-86.

[27] Sakano S, Zhu Y, Sandell LJ. Cartilage-derived retinoic acidsensitive protein and type II collagen expression during fracture healing are potential targets for SOX9 regulation. J Bone Miner Res 1999; 14: 1891-901.

[28] Dudley AC, Khan ZA, Shih SC, et al. Calcification of multipotent prostate tumor endothelium. Cancer Cell 2008; 14: 201-11.

[29] Robbins JC, Akeno N, Mukherjee A, et al. Hypoxia induces chondrocyte-specific gene expression in mesenchymal cells in association with transcriptional activation of SOX9. Bone 2005; 37: 313-22.

[30] De Santa BP, Moniot B, Poulat F, Berta P. Expression and subcellular localization of SF-1, SOX9, WT1, and AMH proteins during early human testicular development. Dev Dyn 2000; 217: 293-8.

[31] Aleman A, Adrien L, Lopez-Serra L, et al. Identification of DNA hypermethylation of SOX9 in association with bladder cancer progression using CpG microarrays. Br J Cancer 2008; 98: 466-73. 